\title{
Resonant interaction between gravitational waves, electromagnetic waves and plasma flows
}

\author{
Martin Servin and Gert Brodin \\ Department of Physics, Umeå University, S-901 87 Umeå, Sweden \\ Accepted for publication in Physical Review D \\ Scheduled issue: 15 August 2003.
}

\begin{abstract}
In magnetized plasmas gravitational and electromagnetic waves may interact coherently and exchange energy between themselves and with plasma flows. We derive the wave interaction equations for these processes in the case of waves propagating perpendicular or parallel to the plasma background magnetic field. In the latter case, the electromagnetic waves are taken to be circularly polarized waves of arbitrary amplitude. We allow for a background drift flow of the plasma components which increases the number of possible evolution scenarios. The interaction equations are solved analytically and the characteristic time scales for conversion between gravitational and electromagnetic waves are found. In particular, it is shown that in the presence of a drift flow there are explosive instabilities resulting in the generation of gravitational and electromagnetic waves. Conversely, we show that energetic waves can interact to accelerate particles and thereby produce a drift flow. The relevance of these results for astrophysical and cosmological plasmas is discussed.
\end{abstract}

PACS numbers:

\section{INTRODUCTION}

In empty and flat space-time linear gravitational wave (GW) perturbations and electromagnetic waves (EMWs) do not interact when propagating in the same direction. If, however, there is a background electromagnetic field or a background curvature present the two waves may couple, interact and exchange energy [1]-[3].

Although GWs typically interact weakly with matter, it is of interest to consider how GW-EMW interaction may be altered by the presence of a magnetized plasma - which is a common state of matter in astrophysical and cosmological scenarios. The interaction may; lead to production or modification of observable EMWs, as considered in the Refs. [4]-[5], energize the plasma by exciting Alfvén and magnetosonic waves [6]-7] which may be of importance in more complex processes such as supernovae explosions, as discussed in Ref. 8]. Furthermore, it may even modify the expected GW signals [9] that are currently under attempted detection and will, hopefully, provides us with a new window through which the universe can be observed. Studying nonlinearly interacting waves may also reveal new types of instabilities that cannot be found using conventional linear stability theory, see e.g. Ref. [10].

Wave interactions are most efficient if they are resonant (coherent), i.e. if the frequencies satisfy certain matching conditions and the relative wave phase remains unchanged for a long time. We will thus not consider incoherent (non-resonant) wave interaction, in which case energy conversion takes place on a much longer time scale. Resonant interaction in vacuum or in the presence of a background electromagnetic field requires only that the frequencies should coincide since both GWs and EMWs propagate along null geodesics and therefore have identical phase velocity equal to $c$, the speed of light. Consider- ing GW-EMW interaction in a "medium" the occurrence of such resonances is more rare because the phase velocity for GW is (in the high frequency approximation and to a very high accuracy) still equal to $c$, whereas for EMWs this occurs only for particular wave frequencies (or in the limit of very high frequencies). For this reason resonant GW-EMW interaction has been studied in Ref. [6] for the case of multiple EMWs, where two or more EMWs that seperately do not propagate along null geodesics together produce perturbations that are resonant with a GW. See also Ref. [11], where generation of GWs with the same mechanism was considered but for interacting sound waves.

Ref. 12 showed that resonant interaction between a GW and a single low-frequency EMW (a "magnetosonic wave", in the terminology of their formalism) can be realized for "incompressible" relativistic magnetofluids, i.e. with sound velocity equal to $c$, but no calculations regarding the coupling strength where made. In Ref. 7] the excitation of magnetosonic waves by GWs was considered with particular focus on almost coherent waves. In Ref. [13] kinetic theory was used to derive, among other things, dispersion relations governing the coupling between a GW and an EMW propagating perpendicular to a background magnetic field. From the dispersion relations in Ref. 13] it is clear that the waves are resonant if the wave frequencies coincide with the electron plasma frequency (defined below) but the strength of the interaction cannot easily be deduced from those results.

In this paper we study the interaction between GWs of small amplitude and EMWs in a magnetized plasma modelled by multifluid equations. In the case of perpendicular propagation with respect to the background magnetic field, we take the EMW to be a high-frequency extraordinary electromagnetic wave (using standard terminology of plasma wave theory), the only wave in this case 
for which we may have phase velocity equal to $c$ (without introducing a more complicated background state). The main focus of our study is on the case of parallel propagation, where we make use of an exact (when neglecting gravitational effects) EMW solution that can be reduced to e.g. high-frequency electromagnetic waves, whistler waves, low-frequency Alfvén waves or waves in electron-positron plasmas. This solution also allows the presence of a relative drift flow of the fluids constituting the plasma. The inclusion of a drift in the background state is important for at least three reasons, when considering GW-EMW perturbations. Firstly, it increases the number of ways that resonant interaction can occur, secondly, it alters the coupling strength between the waves and, most importantly, it supplies the system with free energy. This later fact means, as we will demonstrate, that the background configuration may be unstable, leading to simultaneous generation of GWs and EMWs.

The paper is organized as follows. In section II we give an overview of certain principles of resonant wave interactions. The basic equations are presented in section III, i.e. the Einstein-Maxwell system together with multifluid equations. We demonstrate in section IV that, provided the conditions for the high-frequency approximation are fulfilled, GWs can be taken to be in the transverse traceless gauge also in the presence of matter and we derive the corresponding evolution equations, describing how the GWs are coupled to the matter perturbation. In section $\mathrm{V}$ we derive evolution equations for the EMWs, including the effect of GWs on the EMWs and expressions for the wave energy density. It is shown that circularly polarized EMWs can have negative wave energy for certain background parameter values and simultaneously be resonant with a GW. Resonant interaction between the GWs and EMWs is studied in section VI. Wave interaction equations are derived and their solutions are examined, whereby the characteristic time scales for conversion between gravitational and electromagnetic waves are found. For the case of negative energy EMWs the coupling to GWs give rise to explosive EMW-GW instabilities or, with different initial conditions, acceleration of plasma flow. The results are summarized and further discussed in section VII, including the relevance for astrophysical and cosmological plasmas.

\section{OVERVIEW OF RESONANT WAVE INTERACTIONS}

The natural wave modes of the gravitational field in the high-frequency approximation [16] and of the electromagnetic field in magnetized plasmas [17] are wellknown. Based on these solutions, we choose a set of wave perturbations, $F_{n}$, that we represent with the following complex notation: $F_{n}=f_{n}+$ c.c., where $f_{n}=\mathfrak{f}_{n} e^{i \theta_{(i)}}$, $\mathfrak{f}_{n}$ is the complex amplitude, $\theta_{n}$ the (real) wave phase and c.c. stands for complex conjugation of the preceding term. Letting $f_{1}$ denote the GW (e.g. some linear combi- nation of components of the perturbed metric tensor) and $f_{2}$, say, the electric field of the EMW. From the governing equations, the Einstein-Maxwell-plasma fluid system presented in section III we derive evolution equations for these wave perturbations. As can be found from section IV] and $\nabla$ these equations take the form

$$
\begin{aligned}
& \hat{D}_{1} f_{1}=S_{1}\left(f_{2}, f_{1}\right)+\ldots \\
& \hat{D}_{2} f_{2}=S_{2}\left(f_{1}, f_{2}\right)+\ldots
\end{aligned}
$$

where $\hat{D}_{n}$ is the linear wave propagator and the interaction source term $S_{n}\left(f_{m}, f_{n}\right)$ is an algebraic expression [14] involving $f_{m}$ (and possibly also $f_{n}$ ) such that $S_{n} \equiv \mathfrak{S}_{n} e^{i \phi_{n}}$ is resonant with $f_{n}$, i.e. the relative phase $\theta_{n}-\phi_{n}$ is constant. The dots in Eqs. (11) and (2) indicates that there are in general also terms that are non-resonant with $f_{n}$. Non-resonant terms are discarded, as they only have an effect on a much larger time-scale. The source term $S_{1}$ have the structure such that in the absence of EMWs, $f_{2}=0, S_{1}$ vanishes and Eq. (1) reduces to the free wave equation for GWs, $\left[\partial_{t}^{2}-\partial_{z}^{2}\right] f_{1}=0$. Conversely, if there are no GWs then $S_{2}$ vanishes and Eq. (2) reduces to the plasma EMW equation that corresponds to the assumed perturbation.

Our immediate purpose is to determine the coupling strength and thereby the characteristic time-scales for the wave interactions. We do this by studying spatially uniform monochromatic waves propagating in a (locally) static and uniform background (the relevance for less idealized situations will be discussed in Section (VII). The interaction will then result in time dependent wave amplitudes, governed by the derived evolution equations. Since the source terms are small either proportional to the gravitational coupling constant or to the small GW amplitude - we assume that $\left|\omega_{n} \mathfrak{f}_{n}\right| \gg\left|\partial_{t} \mathfrak{f}_{n}\right|$. This means that, to lowest order, the waves will propagate freely according to the dispersion relation $D_{n}\left(-i \omega_{n}, i \mathbf{k}_{n}\right)=0$ and, to the next level of accuracy, have a slowly evolving amplitude. Applying this, the differential operators in Eqs. (11) and (2) can be approximated by $\hat{D} \approx D(-i \omega, i \mathbf{k})+\left(\partial_{\omega} D\right) \tilde{\partial}_{t}$, where the tilde notation on $\tilde{\partial}_{t}$ means that the derivative acts only on the amplitude. Higher order derivatives are neglected. The evolution equations (11) and (2) thus becomes

$$
\begin{aligned}
& \tilde{\partial}_{t} f_{1}=\left(\partial_{\omega_{1}} D_{1}\right)^{-1} S_{1}\left(f_{2}, f_{1}\right) \\
& \tilde{\partial}_{t} f_{2}=\left(\partial_{\omega_{2}} D_{2}\right)^{-1} S_{2}\left(f_{1}, f_{2}\right)
\end{aligned}
$$

and we refer to these as the interaction equations. Determining $S_{1}$ and $S_{2}$ and studying the solutions of these equations are the main purposes of this paper.

\section{BASIC EQUATIONS}

We take the gravitational and electromagnetic field to be governed by the Einstein field equations

$$
G_{a b}=\kappa T_{a b}
$$


and the Maxwell equations

$$
\begin{aligned}
\nabla_{a} F^{a b} & =j^{b} \\
\nabla_{a} F_{b c}+\nabla_{b} F_{c a}+\nabla_{c} F_{a b} & =0
\end{aligned}
$$

where $G_{a b}$ is the Einstein tensor, $\kappa=8 \pi G, T_{a b}$ is the energy-momentum tensor, $F_{a b}$ is the electromagnetic field tensor, $j^{a}$ is the total four-current density and $\nabla$ denotes covariant derivative. We use units where the velocity of light in vacuum is $c=1$. The metric signature is $(-+++)$ and for the indices we use $a, b, c, \ldots=0,1,2,3$ and $i, j, k, \ldots=1,2,3$. The matter present in the interaction region is a magnetized plasma for which we choose a multifluid description. This means that we take the plasma to consist of a number of interpenetrating charged fluids, one for each species of particles that constitutes the plasma. The fluids interact only through the electromagnetic and gravitational field, i.e. we neglect effects of particle collisions as can be done for most plasmas. The appropriate fluid equations are then, for each fluid component $(i)$, the equation of mass conservation

$$
\nabla_{a}\left(m_{(i)} n_{(i)} u_{(i)}^{a}\right)=0
$$

and the momentum equation

$$
m_{(i)} n_{(i)} u_{(i)}^{a} \nabla_{a} u_{(i)}^{b}=-\nabla^{b} p_{(i)}+q_{(i)} n_{(i)} u_{(i)}^{a} F_{a}^{b}
$$

where $n_{(i)}$ is the proper particle number density, $u_{(i)}^{a}$ is the fluid four-velocity, $m_{(i)}$ is the particle mass, $p_{(i)}$ is the pressure and $q_{(i)}$ is the particle electric charge. For closure this should be supplemented by some equation of state which we do not specify here. The total current density is then given by $j^{a}=\sum_{(i)} q_{(i)} n_{(i)} u_{(i)}^{a}$ and the energy-momentum tensor by $T_{a b}=T_{a b}^{(f l)}+T_{a b}^{(e m)}$, where the fluid contribution is

$$
T_{a b}^{(f l)}=\sum_{(i)}\left(m_{(i)} n_{(i)}+p_{(i)}\right) u_{(i) a} u_{(i) b}+p_{(i)} g_{a b}
$$

and the electromagnetic field contribution is

$$
T_{a b}^{(e m)}=F_{a}^{c} F_{b c}-\frac{1}{4} g_{a b} F^{c d} F_{c d}
$$

From now on we will omit the species index, $(i)$, unless there can be any confusion.

It is practical to rewrite the Maxwell and fluid equations in a more convenient form. In particular we intend to introduce an orthonormal frame so that the interpretation of the quantities becomes more direct. First, we note that by introducing an observer four velocity, $V^{a}$, the electromagnetic field can be decomposed, relative to $V^{a}$, into an electric part, $E^{a}$, and a magnetic part, $B^{a}$, according to $F^{a b}=V^{a} E^{b}-V^{b} E^{a}+\epsilon^{a b c} B_{c}$, where $E_{a}=F_{a b} V^{b}, B_{a}=\frac{1}{2} \epsilon^{a b c} F_{b c}, \epsilon_{a b c}=V^{d} \epsilon_{a b c d}$ and $\epsilon_{a b c d}$ is the 4 dimensional volume element with $\epsilon_{0123}=\sqrt{|\operatorname{det} g|}$. If one chooses an orthonormal frame (in which $V^{a}=(1, \mathbf{0})$ ) with contravariant basis $\left\{\mathbf{e}_{a}\right\}$, the
Maxwell and fluid equations can be formulated as [4], 15]

$$
\begin{aligned}
\nabla \cdot \mathbf{E} & =\rho+\rho_{E} \\
\nabla \cdot \mathbf{B} & =\rho_{B} \\
\mathbf{e}_{0} \mathbf{E}-\nabla \times \mathbf{B} & =-\mathbf{j}-\mathbf{j}_{E} \\
\mathbf{e}_{0} \mathbf{B}+\nabla \times \mathbf{E} & =-\mathbf{j}_{B} \\
\mathbf{e}_{0}(\gamma n)+\nabla \cdot(\gamma n \mathbf{v}) & =\Delta n \\
m n\left(\mathbf{e}_{0}+\mathbf{v} \cdot \nabla\right) \gamma \mathbf{v} & =-\gamma^{-1} \nabla p \\
& +q n(\mathbf{E}+\mathbf{v} \times \mathbf{B})+m n \mathbf{g}
\end{aligned}
$$

where we have introduced an Euclidean three-vector notation $\mathbf{E}=\left(E^{1}, E^{2}, E^{3}\right)$ etc., $\nabla=\left(\mathbf{e}_{1}, \mathbf{e}_{2}, \mathbf{e}_{3}\right), \rho=j^{0}$ and coordinate velocity, $v^{a}$, so that $u^{a}=\gamma v^{a}$ with $\gamma=\left(1-v_{i} v^{i}\right)^{-\frac{1}{2}}$. The effect of the gravitational field is included in the form of effective charges, currents, gravitational forces etc. that were introduced above, and they are given by [15]

$$
\begin{aligned}
\rho_{E} & \equiv-\Gamma_{j i}^{i} E^{j}-\epsilon^{i j k} \Gamma_{i j}^{0} B_{k} \\
\rho_{B} & \equiv-\Gamma_{j i}^{i} B^{j}+\epsilon^{i j k} \Gamma_{i j}^{0} E_{k} \\
\mathbf{j}_{E} & \equiv\left[-\left(\Gamma_{0 j}^{i}-\Gamma_{j 0}^{i}\right) E^{j}+\Gamma_{0 j}^{j} E^{i}\right. \\
& \left.-\epsilon^{i j k}\left(\Gamma_{j 0}^{0} B_{k}+\Gamma_{j k}^{m} B_{m}\right)\right] \mathbf{e}_{i} \\
\mathbf{j}_{B} & \equiv\left[-\left(\Gamma_{0 j}^{i}-\Gamma_{j 0}^{i}\right) B^{j}+\Gamma_{0 j}^{j} B^{i}\right. \\
& \left.+\epsilon^{i j k}\left(\Gamma_{j 0}^{0} E_{k}+\Gamma_{j k}^{m} E_{m}\right)\right] \mathbf{e}_{i} \\
\Delta n & \equiv-\gamma n\left(\Gamma_{0 i}^{i}+\Gamma_{00}^{i} v_{i}+\Gamma_{j i}^{i} v^{j}\right) \\
\mathbf{g} & \equiv-\gamma\left[\Gamma_{00}^{i}+\left(\Gamma_{0 j}^{i}+\Gamma_{j 0}^{i}\right) v^{j}+\Gamma_{j k}^{i} v^{j} v^{k}\right] \mathbf{e}_{i}
\end{aligned}
$$

where $\Gamma_{b c}^{a}$ are the Ricci rotation coefficients.

\section{GRAVITATIONAL WAVE EVOLUTION}

In this section we show that, in the high-frequency approximation 16] for GWs propagating in matter, the GWs can be taken to be in the transverse trace-less (TT) gauge and we give the corresponding evolution equation. In flat space-time linearized gravitational waves can always be taken to be in the TT gauge. In vacuum spacetime with a background curvature this is in general only possible in the high frequency limit, where the ratio of the GW wavelength and the characteristic background length scale (radius of curvature) tends to zero. The advantages of having GWs in the TT-gauge is that the evolution equation becomes more simple, the polarization state is more clear and, most important for us here, it reduces the amount of algebra when calculating the effect on a plasma, e.g. when calculating the gravitational source terms in the EMW evolution equation. However, the (perturbed) energy-momentum tensor should have the same properties as the perturbed Einstein tensor and this will in general not be the case if one just assumes the TT-gauge. Nevertheless, we demonstrate that the TT gauge can also be applied in the presence of matter 
provided the conditions for the high frequency approximation is fulfilled. This result seems to have been overlooked in the litterature.

Let the background gravitational field and the unperturbed plasma fulfill the Einstein field equations, $G_{a b}^{(0)}=\kappa T_{a b}^{(0)}$, and introduce a perturbation so that $G_{a b}=G_{a b}^{(0)}+\delta G_{a b}$ and $T_{a b}=T_{a b}^{(0)}+\delta T_{a b}$. We assume the high frequency approximation so that the background space-time can be taken to be Minkowski space-time 16] and put $g_{a b}=\eta_{a b}+h_{a b}$, where $\eta_{a b}$ is the Minkowski metric and $h_{a b}$ the small metric perturbation that should obey

$$
\delta G_{a b}=\kappa \delta T_{a b}
$$

We limit ourself to linear gravitational perturbations. The perturbed Einstein tensor, linearized in $h_{a b}$, is given by

$$
\delta G_{a b}[\bar{h}]=-\frac{1}{2} \partial^{c} \partial_{c} \bar{h}_{a b}+\partial^{c} \partial_{(b} \bar{h}_{a) c}-\frac{1}{2} \eta_{a b} \partial^{c} \partial^{d} \bar{h}_{c d}
$$

$\bar{h}_{a b} \equiv h_{a b}-\frac{1}{2} \eta_{a b} h$ and the brackets ( ) stands for symmetrization with respect to the enclosed indices. In vacuum, the next step would be to apply the Lorentz gauge condition, $\partial^{b} \bar{h}_{a b}=0$. Instead of this, we split $\bar{h}_{a b}$ according to

$$
\bar{h}_{a b}=\bar{h}_{a b}^{L}+\bar{f}_{a b}
$$

where $\bar{h}_{a b}^{L}$ is the (maximal) part of $\bar{h}_{a b}$ that fulfills the Lorentz gauge condition and the other part, $\bar{f}_{a b}$, containing terms that cannot be fitted into $\bar{h}_{a b}^{L}$ (both terms should be symmetric). With this splitting Eq. (18) reads

$$
-\frac{1}{2} \partial^{c} \partial_{c} \bar{h}_{a b}^{L}+\delta G_{a b}[\bar{f}]=\kappa \delta T_{a b}
$$

Next we assume that the metric perturbation (and the perturbed energy-momentum tensor) is of the form $h_{a b}=$ $\mathfrak{h}_{a b}\left(x^{d}\right) e^{i k_{c} x^{c}}+$ c.c. with the dispersion relation $k_{a} k^{a}=$ 0 . The dependency of $\mathfrak{h}_{a b}$ on $x^{d}$ is assumed weak, i.e. $\left|\partial_{c} \mathfrak{h}_{a b}\right| \ll\left|k_{c} \mathfrak{h}_{a b}\right|$. Computing $\delta G_{a b}[\bar{f}]$ gives to lowest order

$$
\delta G_{a b}[\bar{f}]=-k^{c} k_{(b} \bar{f}_{a) c}+\frac{1}{2} \eta_{a b} k^{c} k^{d} \bar{f}_{c d}
$$

where terms involving slow derivatives (derivatives on the weakly varying amplitude) have been neglected. From this expression it follows, for perturbations with four wave vector $k^{a}=(\omega, 0,0, \omega)$, that $\delta G_{12}[\bar{f}]=\delta G_{21}[\bar{f}]=0$ and $\delta G_{11}[\bar{f}]=\delta G_{22}[\bar{f}]$. Furthermore, nonzero components of $\bar{f}_{a b}$ should be of order $\bar{f} \sim \kappa \delta T / \omega^{2}$ (which must be much smaller than unity in order not to invalidate the linearization in $h_{a b}$ ) for the field equations (21) to be fulfilled.

We now turn to the terms in $\bar{h}_{a b}^{L}$. A gauge transformation $x^{a^{\prime}}=x^{a}+\xi^{a}$ with $\xi^{a} \ll 1$ and $\xi^{a}=-i \tilde{\xi}^{a}\left(x^{c}\right) e^{i k_{b} x^{b}}+$ c.c. alters the metric perturbation by

$$
\bar{h}^{a^{\prime} b^{\prime}}=\bar{h}^{a b}-\tilde{\xi}^{a} k^{b}-\tilde{\xi}^{b} k^{a}+\eta^{a b} \tilde{\xi}^{c} k_{c}
$$

where it is understood that the amplitude, $\tilde{\xi}^{a}\left(x^{c}\right)$, is weakly dependent on $x^{c}$ in the same way as $\bar{h}^{a b}$. We can, however, neglect slow derivatives on $\xi^{a}$ as such terms only produce small corrections to the $\bar{f}_{a b}$ part or, finally in the wave evolution equations, terms of second order slow derivatives. Consequently, $\xi^{a}$ can, as in the vacuum case, be chosen as linear combinations of $\bar{h}_{a b}^{L}$ such that

$$
\bar{h}^{a^{\prime} b^{\prime}}=\bar{h}_{T T}^{a b}+\bar{f}^{a b}
$$

where $\bar{h}_{T T}^{a b}$ is "in the transverse traceless gauge", i.e. $\bar{h}_{T T}^{11}=-h_{T T}^{22} \equiv h_{A}$ and $\bar{h}_{T T}^{12}=\bar{h}_{T T}^{21} \equiv h_{B}$. The evolution equations for $h_{A}$ and $h_{B}$ follows from Eq. (21) by subtracting the 11 and the ${ }_{22}$ components and by - for aestethical reasons only - adding the 12 and 21 components. The result is

$$
\begin{aligned}
& \partial^{c} \partial_{c} h_{A}=-\kappa\left(\delta T_{11}-\delta T_{22}\right) \\
& \partial^{c} \partial_{c} h_{B}=-\kappa\left(\delta T_{12}+\delta T_{21}\right)
\end{aligned}
$$

which depends critically on the fact that $\delta G_{12}[\bar{f}]=$ $\delta G_{21}[\bar{f}]=0$ and $\delta G_{11}[\bar{f}]=\delta G_{22}[\bar{f}]$.

To summarize, for the part of the metric perturbation that represents GWs propagating in the $x^{3}$-direction, $\bar{h}_{T T}^{a b}$, we have derived evolution equations that describes how the waves are coupled to the perturbed energymomentum tensor. Clearly, nonvanishing $\delta T_{11}-\delta T_{22}$ and $\delta T_{12}$ can potentially act to drive or damp GWs. The remaining part of the perturbed metric, given by $\bar{f}_{a b}$, should be included for the sake of consistency. The perturbed energy-momentum that is not accounted for in Eqs. (25) and (26) produces some gravitational response, which is given by $\delta G_{a b}[\bar{f}]$ and found from the remaining components of Eqs. (21) (due to Eqs. (21) and (22) these are purely algebraic equations). As $\vec{f}_{a b}$ represents the self-gravitation of the energy-momentum perturbation it is in general important for the evolution of the matter and electromagnetic field, but since $\bar{f} \sim \kappa \delta T / \omega^{2}$ it is negligible when we consider waves in the high-frequency limit. We conclude that in the highfrequency limit and for slowly evolving wave amplitudes the GWs can be taken to be in the TT-gauge and the metric perturbation $h_{a b}=h_{a b}^{T T}$ evolves according to Eqs. (25) and (26). In this limit the energy density carried by GWs follows directly from the Landau-Lifshitz pseudo energy-momentum tensor 18]

$$
\mathcal{E}_{G W}=\frac{1}{2 \kappa}\left[\left(\partial_{t} h_{A}\right)^{2}+\left(\partial_{t} h_{B}\right)^{2}\right]
$$

When studying the effect of GWs on a magnetized plasma it is practical to use tetrad formalism and introduce an orthonormal frame. A contravariant basis that corresponds to GWs in the TT gauge with propagation in the $x^{3} \equiv z$ direction is given by

$$
\begin{aligned}
& \mathbf{e}_{0}=\partial_{t}, \quad \mathbf{e}_{1}=\left(1-\frac{1}{2} h_{A}\right) \partial_{x}-\frac{1}{2} h_{B} \partial_{y}, \\
& \mathbf{e}_{2}=\left(1+\frac{1}{2} h_{A}\right) \partial_{y}-\frac{1}{2} h_{B} \partial_{x}, \quad \mathbf{e}_{3}=\partial_{z}
\end{aligned}
$$


and the gravitationally induced terms in Eqs. (12)-(17) are (displaying the nonzero terms only)

$$
\begin{aligned}
\mathbf{g} & \left.=-\frac{1}{2} \gamma\left(1-v_{z}\right)\left[v_{x} \partial_{t} h_{A}+v_{y} \partial_{t} h_{B}\right)\right] \mathbf{e}_{1} \\
& -\frac{1}{2} \gamma\left(1-v_{z}\right)\left[v_{x} \partial_{t} h_{B}-v_{y} \partial_{t} h_{A}\right] \mathbf{e}_{2} \\
& -\frac{1}{2} \gamma\left[\left(v_{x}^{2}-v_{y}^{2}\right) \partial_{t} h_{A}+2 v_{x} v_{y} \partial_{t} h_{B}\right] \mathbf{e}_{3} \\
\mathbf{j}_{E} & =-\frac{1}{2}\left[\left(E_{x}-B_{y}\right) \partial_{t} h_{A}+\left(E_{y}+B_{x}\right) \partial_{t} h_{B}\right] \mathbf{e}_{1} \\
& -\frac{1}{2}\left[-\left(E_{y}+B_{x}\right) \partial_{t} h_{A}+\left(E_{x}-B_{y}\right) \partial_{t} h_{B}\right] \mathbf{e}_{2} \\
\mathbf{j}_{B} & =-\frac{1}{2}\left[\left(E_{y}+B_{x}\right) \partial_{t} h_{A}-\left(E_{x}-B_{y}\right) \partial_{t} h_{B}\right] \mathbf{e}_{1} \\
& -\frac{1}{2}\left[\left(E_{x}-B_{y}\right) \partial_{t} h_{A}+\left(E_{y}+B_{x}\right) \partial_{t} h_{B}\right] \mathbf{e}_{2}
\end{aligned}
$$

where we have used $\partial_{z} \approx-\partial_{t}$.

\section{ELECTROMAGNETIC WAVE EVOLUTION}

In this section we present the evolution equations for the EMWs that we are considering. Since the GW fourwave vector is assumed to fulfill the dispersion relation $k_{a} k^{a}=0$, they have phase velocity equal to unity. The EMWs that can interact resonantly with the GWs should then also have phase velocity equal to unity. For simplicity, we restrict our study to the case of perpendicular and parallel propagation to the background magnetic field, respectively.

The plasma is assumed neutral and uniform, with a constant background magnetic field, $B_{(0)}$. The plasma frequency is denoted by $\omega_{p(i)} \equiv\left(n_{(i)} q_{(i)}^{2} / m_{(i)}\right)^{1 / 2}$ and the cyclotron frequency by $\omega_{c(i)} \equiv q_{(i)} B_{(0)} / \gamma_{(i)} m_{(i)}$, where $n_{(i)}$ is the unperturbed proper particle number density. We also allow the presence of a background drift flow in the direction of the magnetic field. The inclusion of the drift flow provides the system with free energy that may be transferred to the GWs and EMWs during the interaction.

In magnetized plasmas there are many electromagnetic wave modes and their properties are well known for flat space-time and for linear (small amplitude) waves (see e.g. [17]). In order to obtain the effect of GWs on the EMWs we derive their evolution equations from the basic equations (12)-(17), including the effect of the gravitational field. For later reference, we note that the total energy density of an EMW is given by

$$
\mathcal{E}_{E M}=\mathbf{E}^{*} \cdot\left(\omega^{-1} \partial_{\omega}\left[\omega^{2} \epsilon\right]\right) \cdot \mathbf{E}
$$

where $\mathbf{E} \propto e^{i(k z-\omega) t}$ is the electric field of the wave, $\omega$ is the frequency and $\epsilon$ is the effective dielectric tensor defined such that $\mathbf{D}=\epsilon \cdot \mathbf{E}$ gives the electric displacement field. For the cold (i.e. zero pressure) linearized plasma fluid equations $\epsilon$ is given by 19$]$

$$
\epsilon=\left(\begin{array}{ccc}
S & -i D & 0 \\
i D & S & 0 \\
0 & 0 & P
\end{array}\right)
$$

where there is a background magnetic field and drift flow $v_{z}$ in the $z$-direction, $S \equiv \frac{1}{2}(R+L), D \equiv \frac{1}{2}(R-L)$ and

$$
\begin{aligned}
& P \equiv 1-\sum_{(i)} \frac{\omega_{p(i)}^{2}}{\left(\omega-k v_{z(i)}\right)^{2}} \\
& R \equiv 1-\sum_{(i)} \frac{\omega_{p(i)}^{2}\left(\omega-k v_{z(i)}\right)}{\omega^{2}\left(\omega-k v_{z(i)}+\omega_{c(i)}\right)} \\
& L \equiv 1-\sum_{(i)} \frac{\omega_{p(i)}^{2}\left(\omega-k v_{z(i)}\right)}{\omega^{2}\left(\omega-k v_{z(i)}-\omega_{c(i)}\right)}
\end{aligned}
$$

Although this result concerns linear waves in a cold plasma it also applies for the large amplitude waves in a moderately warm plasma that we will consider below. This is because they are circularly polarized and purely transverse, making them effectively linear [20] and with no density (nor pressure) perturbations.

\section{A. Perpendicular propagation}

In the case of perpendicular propagation the plasma equations are difficult to treat without resorting to perturbation theory. We restrict ourself in this case to linear perturbations and to this level of approximation the wave perturbations do not produce any effective force, g, that is not rapidly oscillating. This implies that the waves cannot exchange energy with the drift flow in an efficient way. Consequently, we discard the drift flow entirely and only considers one particular wave mode in an electron-ion plasma, namely the extraordinary electromagnetic wave - using standard plasma physics terminology. This is the only wave that can be resonant with a GW in the simplest plasma model, the cold plasma fluid model.

We take the background magnetic field to be $\mathbf{B}_{(0)}=$ $B_{(0)} \mathbf{e}_{1}$. The extraordinary EMW is a high-frequency wave, $\omega \gg \omega_{p i}$ (such that only the electron fluid is perturbed), and has, when propagating in the $z$-direction, the following non-zero linear perturbations: $\delta n, \mathbf{v}=$ $v_{y} \mathbf{e}_{2}+v_{z} \mathbf{e}_{3}, \mathbf{E}=E_{y} \mathbf{e}_{2}+E_{z} \mathbf{e}_{3}$ and $\delta \mathbf{B}=\delta B_{x} \mathbf{e}_{1}$, where the density and velocity perturbation referres to the electron fluid only. For a cold plasma, the Eqs. (12)-(17) reduces to the following equations, linearized in the small perturbations

$$
\begin{aligned}
\partial_{z} E_{z} & =q \delta n \\
\partial_{t} E_{y}-\partial_{z} \delta B_{x} & =-q n v_{y}-j_{E} y \\
\partial_{t} E_{z} & =-q n v_{z} \\
\partial_{t} \delta B_{x}-\partial_{z} E_{y} & =-j_{B} \\
\partial_{t} v_{y} & =\frac{q}{m}\left(E_{y}+v_{z} B_{(0)}\right) \\
\partial_{t} v_{z} & =\frac{q}{m}\left(E_{z}-v_{y} B_{(0)}\right) \\
\partial_{t} \delta n+n \partial_{z} v_{z} & =0
\end{aligned}
$$


which can be combined into the following evolution equation for the wave magnetic field (after one time integration)

$$
\hat{D}_{X} \delta B_{x}=S_{X}
$$

where the wave propagator, $\hat{D}_{X}$, and the source term, $S_{X}$, are

$$
\begin{aligned}
& \hat{D}_{X} \equiv \partial_{t}^{4}+\left(\omega_{p}^{2}+\omega_{h}^{2}\right) \partial_{t}^{2}-\partial_{t}^{2} \partial_{z}^{2}-\omega_{h}^{2} \partial_{z}^{2}+\omega_{p}^{4} \\
& S_{X} \equiv \frac{1}{2} B_{(0)}\left[\partial_{t}^{4}+\left(\omega_{p}^{2}+\omega_{h}^{2}\right) \partial_{t}^{2}+\omega_{p}^{4}\right. \\
&\left.+\partial_{z} \partial_{t}\left(\partial_{t}^{2}+\omega_{p}^{2}+\omega_{c}^{2}\right)\right] h_{A}
\end{aligned}
$$

and we have made use of Eqs. (28)-(30), linearized in the wave perturbations and introduced the hybrid frequency $\omega_{h}^{2} \equiv \omega_{p}^{2}+\omega_{c}^{2}$. In the absence of GWs Eq. (40) reduces to $\hat{D}_{X} \delta B_{x}=0$ and, in particular, for wave perturbations $\delta B_{x} \propto e^{i(k z-\omega t)}$ it produces the dispersion relation for the extraordinary wave

$$
D_{X} \equiv \omega^{4}-\left(\omega_{h}^{2}+\omega_{p}^{2}+k^{2}\right) \omega^{2}+\omega_{h}^{2} k^{2}+\omega_{p}^{4}=0
$$

It should be pointed out that the extraordinary mode, with the given polarization, only couples to $h_{A}$ and not to $h_{B}$. Computing the perturbed energy-momentum tensor gives, linear in the perturbations,

$$
\begin{aligned}
& \delta T_{11}=-\delta T_{22}=-\delta B_{x} B_{(0)} \\
& \delta T_{12}=\delta T_{21}=0
\end{aligned}
$$

The dispersion relation (41) implies that the condition for having phase velocity $\omega / k=1$ is $\omega=\omega_{p}$. We also note that from Eq. (311) and Eqs. (33)-(39) it follows, after some algebra, that the energy density of a free extraordinary wave with $\omega=\omega_{p}$ is given by

$$
\mathcal{E}_{X}=2 \omega_{c}^{-2} \omega_{h}^{2}\left|\delta B_{x}\right|^{2}
$$

\section{B. Parallel propagation}

If one neglects general relativistic effects, there exist exact solutions of the multifluid equations that represents EMWs propagating parallel to a background magnetic field (see e.g. 21]). These solutions describe circularly polarized waves of arbitrary amplitude and arbitrary frequency and can thus, taking the appropriate limits, be reduced to high-frequency electromagnetic waves, whistler waves, low-frequency Alfvén waves or waves in electronpositron plasmas. The solution can also be extended to include a background drift flow 22]. We derive the evolution equation for this wave for a two-component plasma, including the effects of GWs. The indices $e$ and $i$ will now stand for negatively charged particles (e.g. electrons) and positively charged particles (e.g. positive ions), respectively, but we make no assumptions for the mass ratio $m_{e} / m_{i}$. The calculations are therfore equally valid for electron-ion type of plasmas as well as for electronpositron type of plasmas.

We take the background magnetic field to be $\mathbf{B}_{(0)}=$ $B_{(0)} \mathbf{e}_{3}$ and suppose there is a velocity drift, $v_{z}$, in the $z$-direction. The above mentioned circularly polarized EMW, propagating in the $z$-direction, has the following perturbations (of arbitrary amplitude) $\mathbf{v}=v_{x} \mathbf{e}_{1}+v_{y} \mathbf{e}_{2}$, $\mathbf{E}=E_{x} \mathbf{e}_{1}+E_{y} \mathbf{e}_{2}$ and $\delta \mathbf{B}=\delta B_{x} \mathbf{e}_{1}+\delta B_{y} \mathbf{e}_{2}$, where $\mathbf{v}$ and $\delta \mathbf{B}$ are parallel and all perturbations are functions of $z$ and $t$ alone. Note that circular polarization implies that $\gamma$ depends only on the wave amplitude and on $v_{z}$, not on the rapidly varying phase. In the following we treat $\gamma$ as being constant and $v_{z}$ as a constant uniform background flow. Small (linear) deviations in $\gamma$ and $v_{z}$ can still be described (see Section VI and VIII). For convenience we introduce the variables $E_{ \pm}=E_{x} \pm i E_{y}$, and similarly for all other vector variables. In these variables, suitable for circularly polarized waves (the plus/minus variables corresponds to the amplitudes of the right/left hand polarization), those equations of Eqs. (12)-(17) that governs the given perturbations can be rewritten as

$$
\begin{aligned}
\left(\partial_{t}+v_{z} \partial_{z}\right) \gamma v_{ \pm} & =\frac{q}{m}\left(E_{ \pm} \mp i B_{0} v_{ \pm} \pm i v_{z} B_{ \pm}\right)+g_{ \pm} \\
\partial_{t} E_{ \pm} & = \pm i \partial_{z} B_{ \pm}-\Sigma q n \gamma v_{ \pm}-j_{E \pm} \\
\partial_{t} B_{ \pm} & =\mp i \partial_{z} E_{ \pm}-j_{B \pm}
\end{aligned}
$$

where the meaning of $g_{ \pm}$is $g_{ \pm}=g_{x} \pm i g_{y}$ and similarly for $j_{E \pm}$ and $j_{B \pm}$. Recall that there are two sets of Eqs. (45)(47), one set for each particle species. These equations imply the following evolution equation for $E_{ \pm}$

$$
\hat{D}_{E} E_{ \pm}=S_{E}
$$

where the wave propagator is

$$
\begin{aligned}
\hat{D}_{E} & \equiv \hat{G}_{e} \hat{G}_{i} \square+\omega_{p i}^{2} \hat{G}_{e} \hat{d}_{i}+\omega_{p e}^{2} \hat{G}_{i} \hat{d}_{e} \\
\square & \equiv \partial_{t}^{2}-\partial_{z}^{2} \\
\hat{d}_{(i)} & \equiv \partial_{t}+v_{z(i)} \partial_{z} \\
\hat{G}_{(i)} & \equiv \partial_{t}+v_{z(i)} \partial_{z} \pm i \omega_{c(i)}
\end{aligned}
$$

and the source term is

$$
\begin{aligned}
S_{E} & \equiv-q_{i} n \hat{G}_{e} \partial_{t} g_{i \pm}-q_{e} n \hat{G}_{i} \partial_{t} g_{e \pm} \\
& \pm i\left(\omega_{p i}^{2} v_{i z} \hat{G}_{e}+\omega_{p e}^{2} v_{e z} \hat{G}_{i}-\hat{G}_{e} \hat{G}_{i} \partial_{z}\right) j_{B \pm} \\
& -\hat{G}_{e} \hat{G}_{i} \partial_{t} j_{E \pm}
\end{aligned}
$$

In the absence of the gravitational source terms the evolution equation (48) reduces to $\hat{D}_{E} E_{ \pm}=0$ and for this 
case we in particular note the solution [21]

$$
\begin{aligned}
E_{ \pm} & =E e^{i(k z-\omega t)} \\
B_{ \pm} & = \pm i \frac{k}{\omega} E_{ \pm} \\
v_{ \pm} & =i \Lambda E_{ \pm} \\
\Lambda & \equiv \frac{q}{\gamma m} \frac{\omega-k v_{z}}{\omega\left(\omega-k v_{z} \mp \omega_{c}\right)} \\
\gamma & =\left(1-v_{ \pm} v_{\mp}-v_{z}^{2}\right)^{-\frac{1}{2}}
\end{aligned}
$$

with the dispersion relation

$$
\begin{aligned}
0 & =D_{E} \equiv\left(\omega-k v_{z e} \mp \omega_{c e}\right)\left(\omega-k v_{z i} \mp \omega_{c i}\right)\left(\omega^{2}-k^{2}\right) \\
& -\omega_{p i}^{2}\left(\omega-k v_{z e} \mp \omega_{c e}\right)\left(\omega-k v_{z i}\right) \\
& -\omega_{p e}^{2}\left(\omega-k v_{z i} \mp \omega_{c i}\right)\left(\omega-k v_{z e}\right)
\end{aligned}
$$

that can be written more compact as

$$
\omega^{2}-k^{2}=\sum_{(i)} \frac{\omega_{p(i)}^{2}\left(\omega-k v_{z(i)}\right)}{\omega-k v_{z(i)} \mp \omega_{c(i)}}
$$

We again emphasize that the solution is valid for arbitrarily large amplitudes. The condition for the plasma to be electrically neutral, $j^{0}=0$, implies $\gamma_{e} n_{e}=\gamma_{i} n_{i}$. As $\gamma_{(i)} n_{(i)}$ is a preserved quantity (by Eq. (16)), the proper density, $n_{(i)}$, is not. Accordingly, both $\omega_{p(i)}$ and $\omega_{c(i)}$ depend on $\gamma_{(i)}$. This makes the dispersion relation amplitude dependent.

For future reference we devote the remainder of this section to establish some properties of the free EMWs. As we will focus on EMWs with $\omega=k$ we note that the dispersion relation in this case implies the following relation between the background magnetic field and the drift velocities

$$
\Omega_{c e}= \pm\left(\gamma_{e}+\frac{m_{i}}{m_{e}} \gamma_{i}\right) \frac{\left(1-v_{z e}\right)\left(1-v_{z i}\right)}{v_{z e}-v_{z i}}
$$

where $\Omega_{c e} \equiv \gamma_{e} \omega_{c e} / \omega=\omega^{-1} q_{e} B_{(0)} / m_{e}$ is the normalized (and preserved) "electron" cyclotron frequency and we have made use of the neutrality condition $\gamma_{e} n_{e}=\gamma_{i} n_{i}$. Note that for any given background plasma parameters (except the absence of any drifts), there always exist a wave frequency fulfilling Eq. (58). The components of the perturbed energy-momentum tensor, for this large amplitude circularly polarized EMW, that couples to GWs has the values

$$
\begin{aligned}
& \delta T_{11}=-\frac{1}{2}\left(E_{x}^{2}+B_{x}^{2}-E_{y}^{2}-B_{y}^{2}\right)+\sum \gamma m n v_{x}^{2} \\
& \delta T_{22}=\frac{1}{2}\left(E_{x}^{2}+B_{x}^{2}-E_{y}^{2}-B_{y}^{2}\right)+\sum \gamma m n v_{y}^{2} \\
& \delta T_{12}=-\left(E_{x} E_{y}+B_{x} B_{y}\right)+\sum \gamma m n v_{x} v_{y}
\end{aligned}
$$

Because the wave is circularly polarized and transversal it is effectively of linear nature (the Lorentz force is linear in the wave amplitude) and has no density (nor pressure) perturbations. Therefore, the wave energy density can be calculated by means of Eq. (31). For the given wave perturbations, the wave energy density is given by

$$
\begin{aligned}
& \mathcal{E}_{E}=\frac{1}{2 \omega} \partial_{\omega}\left(\omega^{2} A_{ \pm}\right)\left|E_{ \pm}\right|^{2} \\
& A_{ \pm} \equiv 1-\sum \frac{\omega_{p}^{2}}{\omega^{2}} \frac{\omega-k v_{z}}{\omega-k v_{z} \mp \omega_{c}}
\end{aligned}
$$

Applying the dispersion relation (57) with $\omega=k$, the wave energy density can, after some algebra, be formulated as

$$
\begin{aligned}
\mathcal{E}_{E} & =\frac{1}{2 \omega^{2} \alpha_{e} \alpha_{i}}\left[2 \omega^{2} \alpha_{e} \alpha_{i}-\omega_{p e}^{2}\left(1-v_{e}+\alpha_{i}\right)\right. \\
& \left.-\omega_{p i}^{2}\left(1-v_{i}+\alpha_{e}\right)\right]\left|E_{ \pm}\right|^{2}
\end{aligned}
$$

where we have introduced the abbreviation

$$
\alpha_{(i)} \equiv 1-v_{(i)} \mp \omega_{c(i)} / \omega
$$

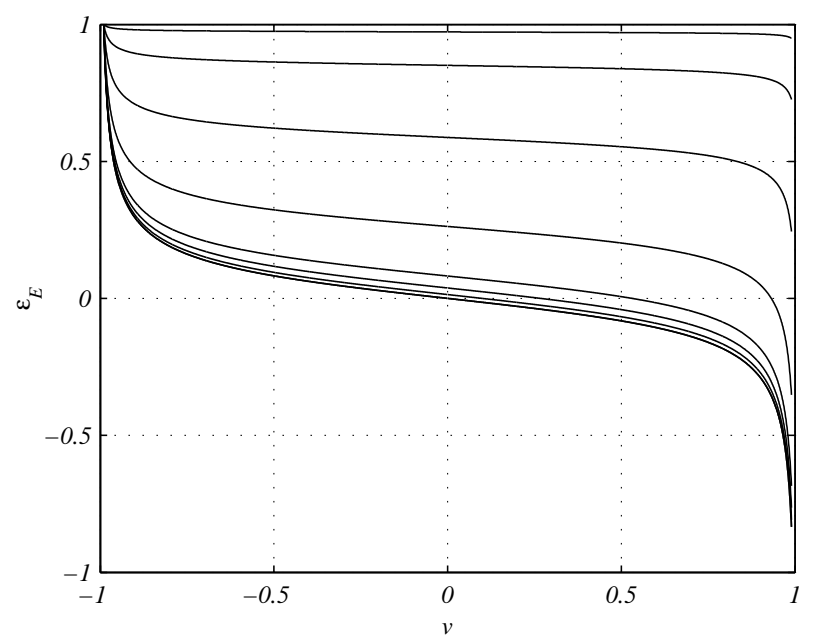

FIG.1. The dependence of wave energy $\mathcal{E}_{E}$ (normalized by $\left|E_{ \pm}\right|^{2}$ and rescaled) on drift flow $v \equiv v_{i}=-v_{e}$ for null geodesic small amplitude waves in an electron-ion plasma $\left(m_{e} / m_{i} \sim 1000\right)$. The different curves, from the top and down, corresponds to the plasma frequencies $\Omega_{p e}=2,5,10,20,40,60,100,1000$. As the sign of the energy is the main focus here, the energy in the different cases are rescaled by suitable factors.

It is important to note here that $\mathcal{E}_{E}$ is, in contrast to $\mathcal{E}_{G W}$ and $\mathcal{E}_{X}$, not positive definite. For some background parameters the wave energy density may be negative [10]. This is due to the presence of the velocity drifts, providing the system with free energy. Negative energy waves are wave perturbations such that the total energy of the plasma is less than the energy of the unperturbed state [23]. To show that there are waves fulfilling $\omega=k$ and $\mathcal{E}_{E}<0$ simultaneously, we consider an electron-ion plasma $\left(m_{e} / m_{i} \sim 1000\right)$ with drift flows $v_{e}=-v_{i}$. In FIG 1 the wave energy factor $\mathcal{E}_{E} /\left|E_{ \pm}\right|^{2}$ (for small amplitude waves) is presented, as a function of ion 
drift velocity, for a number of different values of the normalized "electron" plasma frequency $\Omega_{p e} \equiv \gamma_{e} \omega_{p e} / \omega$ and with the cyclotron frequency fulfilling the condition (58). As the sign of the energy is the main focus here, the energy factor in the different cases are rescaled by suitable factors. Negative wave energy occurs for positive ion velocities [24]. The minimum required ion velocity depends inversely on the plasma frequency. For $\Omega_{p e}=1000$, negative wave energy requires only $v_{i} \gtrsim 10^{-4}$ whereas for $\Omega_{p e} \lesssim 20$ it requires highly relativistic velocities, $v_{i} \gtrsim 0.95$.

\section{RESONANT WAVE INTERACTION}

The wave evolution equations (25), (26), (40) and (48) where derived with no assumptions on the specific wave forms. In this section we assume the wave frequencies and the (uniform) background plasma to be in a state where wave resonance occurs, since this gives the most efficient energy transfer, and we derive the corresponding wave interaction equations. We let

$$
\begin{aligned}
h_{A, B} & =\tilde{h}_{A, B}+c . c . \\
\delta B_{x} & =\tilde{B}+c . c . \\
E_{ \pm} & =E_{x} \pm i E_{y}
\end{aligned}
$$

with $\tilde{h}_{A, B}=\mathfrak{h}_{A, B} e^{i k_{G a} x^{a}}, \tilde{B}=\mathfrak{B} e^{i k_{X} x_{a} x^{a}}$ and $E_{ \pm}=$ $\mathfrak{E} e^{i k_{E} a^{x^{a}}}$ and the four-wave vectors $k_{G}^{a}=\left(\omega_{G}, 0,0, k_{G}\right)$, $k_{X}^{a}=\left(\omega_{X}, 0,0, k_{X}\right)$, and $k_{E}^{a}\left(\omega_{E}, 0,0, k_{E}\right)$ are assumed to fulfill the dispersion relations $k_{G a} k_{G}^{a}=0$, Eq. (41) and Eq. (57), respectively. As before, c.c. stands for the complex conjugate of the preceding term. The conditions for the EMWs to be resonant with a GW are, in the case of perpendicular propagation

$$
k_{G}^{a}=k_{X}^{a}
$$

and in the case of parallel propagation

$$
k_{G}^{a}=2 k_{E}^{a}
$$

Here we have used that the extraordinary EMW contributes linearly to the perturbed energy momentum tensor (42) and the circular polarized large amplitude EMW quadratically in (59)- (61) and thus produces terms proportional to $e^{i 2\left(k_{E} z-\omega_{E} t\right)}$. Consistently, the source terms for the extraordinary EMW is linear (in the GW amplitude) whereas for the circular polarized large amplitude EMW there are terms proportional to $E_{ \pm} h_{A, B}$. Since (in the high frequency limit) $\omega_{G} / k_{G}=1$, also the EMWs should have $\omega_{X} / k_{X}=1$ and $\omega_{E} / k_{E}=1$ in order to remain in phase with the GW. The interaction is weak in the sense that the coupling terms in the evolution equations (25), (26), (40) and (48) are either proportional to the small GW amplitude or the gravitational coupling constant. This leads to a weak time dependence in the wave amplitudes $\mathfrak{h}, \mathfrak{B}$ and $\mathfrak{E}$, i.e. $\partial_{t} \mathfrak{h} \ll \omega_{G} \mathfrak{h}$ etc. We will use the notation of "slow derivatives", $\tilde{\partial}_{t}$, that acts only on the wave amplitude, so that e.g. $\partial_{t} h_{A}=i \omega_{G} h_{A}+\tilde{\partial}_{t} h_{A}$, and we will neglect slow derivatives that are of second or higher order. Consistent with this level of approximation, we make use of the relations between the perturbations valid for free waves, i.e. Eqs (51)-(53), in the computation of the source terms. Corrections to this approximations produces higher order source terms.

We now treat the two cases of propagation direction separately.

\section{A. Perpendicular propagation}

From the dispersion relation (41) it follows that in order for the extraordinary EMW to propagate along null geodesics the wave frequencies must be equal to the plasma frequency, $\omega \equiv \omega_{p e}$. Although this is a special case, a GW or an EMW propagating in a slowly varying background density may often reach such resonance regions. Assuming resonant waves, we take $k_{G}^{a}=$ $k_{X}^{a}=(\omega, 0,0, \omega)$. The wave evolution equations (25) and (40) then imply the following wave interaction equations (dropping the tilde notation on the waves and denoting $h \equiv h_{A}$ and $\left.B \equiv \delta B\right)$

$$
\begin{aligned}
\tilde{\partial}_{t} h & =-i C_{G} B \\
\tilde{\partial}_{t} B & =-i C_{X} h
\end{aligned}
$$

where $C_{G} \equiv \omega^{-1} \kappa B_{(0)}$ and $C_{X} \equiv \frac{1}{2} \omega \omega_{h}^{-2} \omega_{c}^{2} B_{(0)}$. From Eqs. (27) and (44) the total wave energy is $\mathcal{E}_{G W}+\mathcal{E}_{X}=$ $\omega^{2} \kappa^{-1}|h|^{2}+2 \omega_{c}^{-2} \omega_{h}^{2}|B|^{2}$ and it follows from Eq. (67) and (68) that this is a conserved quantity. The interaction equations has the following solution for the wave amplitudes

$$
\begin{aligned}
B & =B_{0} \cos (\psi t)+i C_{X} \psi^{-1} h_{0} \sin (\psi t) \\
h & =h_{0} \cos (\psi t)+i C_{G} \psi^{-1} B_{0} \sin (\psi t)
\end{aligned}
$$

where $B_{0} \equiv B(t=0)$ and $h_{0} \equiv h(t=0), \psi \equiv \sqrt{C_{X} C_{G}}=$ $\sqrt{\frac{\kappa}{2}}\left|\omega_{h}^{-1} \omega_{c} B_{(0)}\right|$. The solution shows that the total wave energy alternates between the GW and the EMW with the frequency $\psi$. In most applications $\psi$ is very small and it is then meaningful to linearize the trigonometric functions in $\psi$ and it is clear that, for time scales smaller than $\psi^{-1}$, GWs are converted into EMWs as $h=C_{G} B_{0} t$ or, alternatively, EMWs are converted into GWs as $B=$ $C_{X} h_{0} t$. It should be noted that in the later case the time $t_{n l}$ for the EMW perturbation to reach the nonlinear stage, $B \sim B_{(0)}$, is typically much smaller than total energy conversion time, $\psi^{-1}$.

\section{B. Parallel propagation}

For resonant interaction between EMWs and GWs in the parallel case we take $k_{G}^{a}=2 k_{E}^{a}=(2 \omega, 0,0,2 \omega)$. As 
the EMWs that we are considering are circularly polarized waves, we also introduce variables for circularly polarized GWs $h_{ \pm} \equiv h_{A} \pm i h_{B}$. If the GW and the EMW are oppositely polarized the coupling vanishes, so from here on we assume identical polarization. The evolution Eqs. (25) and (26) combines to

$$
\left(-\partial_{t}^{2}+\partial_{z}^{2}\right) h_{ \pm}=-\sum \kappa m n \gamma^{2} v_{ \pm}^{2}
$$

and together with the EMW evolution equation (48) this implies the following interaction equations for the variables $h \equiv h_{ \pm}$and $E \equiv E_{ \pm}$

$$
\begin{aligned}
\tilde{\partial}_{t} h & =i C_{G W} E^{2} \\
\tilde{\partial}_{t} E & =i C_{E} E^{*} h
\end{aligned}
$$

where

$$
\begin{aligned}
C_{G W} & \equiv \frac{\kappa}{4 \omega} \sum m n \gamma^{2} \Lambda^{2} \\
C_{E} & \equiv \omega^{2}\left[\gamma_{i} \frac{m_{i}}{q_{i}} \Lambda_{i} \omega_{p i}^{2}\left(1-v_{z i}\right) \alpha_{e}\right. \\
& \left.+\gamma_{e} \frac{m_{e}}{q_{e}} \Lambda_{e} \omega_{p e}^{2}\left(1-v_{z e}\right) \alpha_{i}\right] \\
& \times\left[2 \omega^{2} \alpha_{e} \alpha_{i}-\omega_{p i}^{2}\left(1-v_{z i}+\alpha_{e}\right)\right. \\
& \left.-\omega_{p e}^{2}\left(1-v_{z e}+\alpha_{i}\right)\right]^{-1}
\end{aligned}
$$

In the derivation we have noted that the effective currents, $\mathbf{j}_{E}$ and $\mathbf{j}_{B}$, vanishes and used that $g_{ \pm}=-\frac{1}{2} \gamma(1-$ $\left.v_{z}\right) v_{ \pm}^{*} \partial_{t} h_{ \pm}$. The coupling coefficients can be related to the wave energy densities, Eqs. (27) and (63), according to

$$
\begin{aligned}
C_{G W} & =\mathcal{C} \mathcal{E}_{G W}^{-1}|h|^{2} \\
C_{E} & =\mathcal{C} \mathcal{E}_{E}^{-1}|E|^{2}
\end{aligned}
$$

where

$$
\mathcal{C} \equiv \frac{1}{2 \omega} \sum \frac{\omega_{p(i)}^{2}}{\alpha_{(i)}^{2}}\left(1-v_{z(i)}\right)^{2}
$$

It is then straightforward to confirm that Eqs. (72) and (73) implies that the total wave energy density

$$
\begin{aligned}
\mathcal{E} & =\frac{2 \omega^{2}}{\kappa}|h|^{2}+\frac{1}{2 \omega^{2} \alpha_{e} \alpha_{i}}\left[2 \omega^{2} \alpha_{e} \alpha_{i}\right. \\
& \left.-\omega_{p i}^{2}\left(1-v_{z i}+\alpha_{e}\right)-\omega_{p e}^{2}\left(1-v_{z e}+\alpha_{i}\right)\right]|E|^{2}
\end{aligned}
$$

is conserved.

The solution to the wave interaction equations (72) and (73) can be given in terms of Jacobi elliptic functions. We exploit the fact that the system can be reformulated as a three-wave interacting system (see e.g. [10]) with two identical EMWs and one GW. This is done by taking $E_{2}=E_{3} \equiv \frac{1}{2} E$ such that Eq. (73) can be splitted into two (identical, but differently labeled) equations and the right hand side of Eq. (72) reads $i 4 C_{G W} E_{2} E_{3}$. Next we convert the obtained three-wave interaction equations to a real system and renormalize the amplitudes such that the coupling coefficients become unity. The real system is 25] (dropping the tilde notation)

$$
\begin{aligned}
& \partial_{t} \psi_{1}=s_{1} c_{1} \psi_{2} \psi_{3} \cos (\phi) \\
& \partial_{t} \psi_{2}=s_{2} c_{2} \psi_{1} \psi_{3} \cos (\phi) \\
& \partial_{t} \psi_{3}=s_{3} c_{3} \psi_{1} \psi_{2} \cos (\phi)
\end{aligned}
$$

where we have taken $i h=\psi_{1} e^{i \phi_{1}}, E_{n}=\psi_{n} e^{i \phi_{n}}$, $-4 C_{G W}=s_{1} c_{1}, C_{E}=s_{n} c_{n}($ for $n=2,3)$ with $\psi_{1}=|h|$, $\psi_{n}=\left|E_{n}\right|, c_{1}=\left|C_{G W}\right|, c_{n}=\left|C_{E}\right|, \phi=\phi_{1}-\phi_{2}-\phi_{3}$ and, $s_{1}, s_{2}$ and $s_{3}$ are the signs of $-C_{G W}$ and $C_{E}$, respectively. The coupling coefficients $c_{1}, c_{2}$ and $c_{3}$ are made unity by the renormalization

$$
\begin{aligned}
& \psi_{1} \rightarrow \Psi_{1} \equiv \sqrt{c_{2} c_{3}} \psi_{1} \\
& \psi_{2} \rightarrow \Psi_{2} \equiv \sqrt{c_{3} c_{1}} \psi_{2} \\
& \psi_{3} \rightarrow \Psi_{3} \equiv \sqrt{c_{1} c_{2}} \psi_{3}
\end{aligned}
$$

and we also note that we may, with no loss in generality, assume $s_{2}=s_{3}=1$ (it is only the sign of $s_{1} s_{2}=s_{1} s_{3}$ that matters because an overall sign can be removed by a renormalization). The general solution can then be found in litterature, e.g. in Ref. [10]. The strongest coupling occurs for $\cos \phi= \pm 1$ and in this case there are some particularly simple solutions that are listed below (recall that $\left.\Psi_{2}=\Psi_{3}\right)$.

a) EMW to GW conversion with positive EMW energy

$$
s_{1}=-1, \Psi_{1}(0)=0, \Psi_{2,3}(0)=\Psi \text { and } \cos \phi=-1
$$

$$
\Psi_{1}=\Psi \tanh (\Psi t) \quad \Psi_{2,3}=\Psi \operatorname{sech}(\Psi t)
$$

b) EMW-GW instability with no initial GW

$$
\begin{gathered}
s_{1}=1, \Psi_{1}(0)=0, \Psi_{2,3}(0)=\Psi \text { and } \cos \phi=1 \\
\Psi_{1}=\Psi \tan (\Psi t) \quad \Psi_{2,3}=\Psi \sec (\Psi t)
\end{gathered}
$$

c) EMW-GW instability with equal initial amplitudes

$$
\begin{gathered}
s_{1}=1, \Psi_{1}(0)=\Psi_{2,3}(0)=\Psi \text { and } \cos \phi=1 \\
\Psi_{1}=\left(\Psi^{-1}-t\right)^{-1} \quad \Psi_{2,3}=\left(\Psi^{-1}-t\right)^{-1}
\end{gathered}
$$

d) EMW-GW interaction with decaying amplitudes

$$
\begin{gathered}
s_{1}=1, \Psi_{1}(0)=\Psi_{2,3}(0)=\Psi \text { and } \cos \phi=-1 \\
\Psi_{1}=\left(\Psi^{-1}+t\right)^{-1} \quad \Psi_{2,3}=\left(\Psi^{-1}+t\right)^{-1}
\end{gathered}
$$


Also conversion from GW to EMW energy occurs (given that there is a small initial EMW perturbation) the solution is somewhat more complicated than the ones listed above [10]. Note that $\Psi_{1}(0)=\Psi$ and $\Psi_{2,3}(0)=0$ only has the trivial solution $\Psi_{1}=\Psi$ and $\Psi_{2,3}=0$. The solution a) corresponds to an EMW being converted into a GW on the characteristic time scale $\pi / 2 \Psi$. The solutions b) and c) are explosive solutions, i.e. the wave amplitudes diverges on a finite time $t=\pi / 2 \Psi$ and $t=1 / \Psi$, respectively. These explosive instabilities can only occur for $s_{1}=s_{2}=s_{3}$, which is equivalent to saying that the GW and EMW energy must be of different sign. The explosive instability thus relies on the existence of negative energy EMWs and it is the free energy connected with the background drift flow that feeds the instability. We return to these interesting cases in section VII The solution d) also involves a negative energy EMW, but in this case both the EMW and the GW amplitudes tends to zero as $\left(\Psi^{-1}+t\right)^{-1}$. This means that wave energy is converted into free energy of the background state, i.e. acceleration of the drift flow. As for the cases when the wave phases are not fulfilling $\cos \phi= \pm 1$ the behaviour is similar [25] but with somewhat larger characteristic times.

We now explore the possibility, indicated by the solution $d$ ), that the waves may interact to accelerate the background drift flow. For the wave perturbations we are considering here, the fluid equation of motion (17) has the property $v_{i} \partial_{t} \gamma v^{i}=g_{z}$ (where $\left.i=1,2,3\right)$. This implies that $\xi \equiv \gamma\left(1-v_{z}\right)$ is an exactly conserved quantity, as is seen by making the computation

$$
\partial_{t} \xi \equiv \partial_{t} \gamma-\partial_{t} \gamma v_{z}=v_{i} \partial_{t} \gamma v^{i}-g_{z}=0
$$

Making use of this, the $z$-component of the fluid momentum equation (Eq. (17)) may be rewritten as

$$
\begin{aligned}
\partial_{t} v_{z} & =\frac{i \omega}{2} \xi \Lambda^{2}\left(E^{2} h^{*}-E^{* 2} h\right) \\
& =-\omega \xi \Lambda^{2}|E|^{2}|h| \cos (\phi)
\end{aligned}
$$

where the phase angle $\phi$ has the same meaning as in Eqs. (80)-(82). From this equation it is clear that the drift flow may be accelerated or decellerated in the $z$-direction by the interacting waves. Maximum acceleration occurs for $\cos \phi=-1$. In the pump-wave approximation, where the waves are assumed energetic and can be taken to be of constant amplitude (an approximation breaking down when the energy of the drift flow becomes comparable with the wave energies), the drift flow $v_{z}$ grows linearly in time as (for $\cos \phi=-1$ )

$$
v_{z}=v_{z}(0)+\omega \xi \Lambda^{2}|E|^{2}|h| t
$$

and we point out that for relativistic EMW amplitudes $\Lambda^{2}|E|^{2}=\left|v_{ \pm}\right|^{2} \approx 1$.

\section{SUMMARY AND DISCUSSION}

In this paper we have studied the interaction between GWs and EMWs in magnetized plasmas for perpendicular and parallel propagtion with respect to the background magnetic field. The wave evolution equations were derived in section [V] and $\mathrm{V}$ An important point here which, as far as we know, has not been fully recognized previously is that, given the high frequency approximation, the GWs can be taken to be in the TT gauge even in the presence of matter. Consequently, the deduction of the GW source term associated with the EMW perturbed energy-momentum tensor is simplified, see Eqs. 25) and (26). The GW, on the other hand, produces effective currents in the Maxwell equations and an effective gravitational force on the plasma, resulting in a source term in the EMW evolution equations (40) and (48). Furthermore, it was shown in section $\nabla$ that, provided there is a velocity drift in the background state, the wave energy may be negative 10] for EMWs with phase velocity equal to $c=1$ (when propagating parallel to the background magnetic field).

Resonant interaction (involving a single EMW) may occur when the frequencies matches and the phase velocity of the EMW coincide with that of the GW, which to a very good precision equals $c=1$ in the high-frequency approximation. The wave interaction equations governing the resonant interaction process were derived and analyzed in section VI for the cases of perpendicular (highfrequency extraordinary EMWs) and parallel propagation (finite amplitude circular polarized EMWs) with respect to the background magnetic field. In both cases the interaction equations were shown to imply conservation of total wave energy.

i) Perpendicular propagation. The wave interaction equations for this case are Eqs. (67) and (68). The solution, given by Eqs. (69) and (70), reveals that the conversion rate [26] is of order $\psi=(\kappa / 2)^{1 / 2} \omega_{c} \omega_{h}^{-1} B_{(0)}$. The effect of the plasma is to diminish the coupling strength. The conversion rate becomes $\psi=(\kappa / 2)^{1 / 2} B_{(0)}$ for strong magnetic fields, i.e. $\omega_{c} \gg \omega_{p}$ (similar to having no plasma present), whereas for weak magnetic fields, $\omega_{c} \ll \omega_{p}$, the conversion rate is a factor $\omega_{c} / \omega_{p}$ smaller. Although $\psi$ is typically small, GWs may still generate EMWs with significant amplitude. For small $\psi t$, the EMW are converted into GWs as $B=\frac{1}{2} \omega_{p} \omega_{c}^{2} \omega_{h}^{-2} B_{(0)} h_{0} t$.

ii) Parallel propagation with positive $E M W$ energy. The solutions of the wave interaction equations (72) and (73) were found by reformulating them as a three-wave interacting system (with two identical EMWs) for which the general solutions are well known. From the solution (86) it follows that the conversion rate [26] for conversion from EMW to GW is $\Psi=\sqrt{C_{G W} C_{E}}\left|E_{i n i}\right|$, where $E_{i n i}$ is the initial electric field amplitude. The conversion rate increases with increasing initial amplitude and with increasing drift velocity (through $\gamma$ ). In order to have more transparent formulas we consider a special case, namely low-frequency waves, such that $\omega \ll \omega_{c i}, \omega_{c e}$. The conversion rate can be formulated as

$$
\Psi=\left(C_{G W} C_{E}^{2} \mathcal{C}^{-1} \mathcal{E}_{E, i n i}\right)^{\frac{1}{2}}
$$


and in the case of low-frequency waves

$$
\begin{aligned}
C_{G W} & =\frac{\kappa}{2 \omega^{2}} \mathcal{C} \\
C_{E} & =\mathcal{C D}^{-1} \\
\mathcal{E}_{E} & =\mathcal{D}\left|E_{ \pm}\right|^{2}
\end{aligned}
$$

where

$$
\mathcal{C}=\frac{\omega}{2} \sum \frac{\omega_{p}^{2}}{\omega_{c}^{2}}\left(1-v_{z}\right)^{2}, \quad \mathcal{D}=1 \pm \frac{1}{2 \omega} \sum \frac{\omega_{p}^{2}}{\omega_{c}}
$$

and the final GW amplitude is given by $h_{\text {final }}=$ $\left(\mathcal{E}_{E, i n i} \kappa / 2 \omega^{2}\right)^{1 / 2}$.

iii) Parallel propagation with negative EMW energy. As was remarked previously, the EMW energy density (given by Eq. [63) ) may be negative. Such waves are perturbations with the property that the energy of the perturbed system is less than for the unperturbed system [23]. As is clear from FIG 1, this occurs most commonly for low-frequency waves in electron-ion plasmas, i.e. $\omega \ll \gamma_{e} \omega_{p e}$. The wave energy conservation then allows simultaneous growth of the GW and EMW amplitudes. The physical interpretation is that the background flow is unstable with respect to this type of perturbations and the energy associated with the flow is converted into wave energy. Given that there is an ion flow in the direction of wave propagation and a large enough plasma frequency, this instability will always produce waves with a unique EMW frequency determined by Eq. (58) and GW frequency given by the matching condition (66). It should be noted that this is a nonlinear instability and would not have been found using conventional linear stability analysis. The solutions (87) and (88) shows that the background is explosively unstable with respect to GW-EMW perturbations, the amplitudes reaches infinity in a finite time $t \sim \Psi^{-1}$ (the interaction equations are of course invalidated before this time is reached). The solution (89) is an example of the inverse process, where both waves decreases in amplitude. This means that wave energy is converted into free energy of the background state, i.e. the drift flow is accelerated. An evolution equation for the background flow was also derived, see Eq. (91). The response of the plasma on the interacting waves is an acceleration (if $\cos \phi>0$ ) or deceleration (if $\cos \phi<0$ ). The conclusion is that GWs and EMWs can interact to produce a drift flow along the background magnetic field with initial linear growth rate as large as $\omega \gamma\left(1-v_{z}\right)|h|$. The magnitude of the produced drift flow can be estimated from the initial wave energies and the time scale for all the wave energy to be converted into drift flow is at least of the order given by Eq. (89), $t \sim \Psi^{-1}$.

Finally we discuss the relevance of the results presented in this paper to various astrophysical and cosmological processes. The phenomena studied in this paper involve energy transfer between GWs, EMWs and a background flow. This could in principle be an important feature in any scenario involving magnetized plasmas, energetic GWs and EMWs and/or strong background plasma flows, e.g. supernova explosions, gamma-ray bursts, jets of accreting condensed objects, phenomena in the vicinity of neutron stars and in the primordial fluctuations of the cosmological plasma. The energy conversion must take place within reasonable times and/or volumes for the considered processes to be of significance. As a specific numerical example let us first consider graviational to electromagnetic conversion for perpendicular propagation. For the case of GWs from a binary pulsar close to collapse (corresponding to a wavelength of, let us say, $\lambda \sim 10^{5} \mathrm{~m}$ ) we may have $h \sim 0.001$ a few wavelengths away from the pulsar. A rough estimation based on Eq. (69) then gives a distance $L \sim \lambda / h \sim 10^{8} \mathrm{~m}$ for the induced magnetic field to be comparable to the unperturbed field [28]. A significant transfer of GWenergy is not realistic in this example, however, since the background energy density is too low. As another example, let us consider EMW to GW conversion for parallell propagation and positive wave energy in the low-frequency regime. Let us take $B_{0} \sim 10^{-3} \mathrm{~T}$ and $n_{0} \sim 10^{18} \mathrm{~m}^{-3}$. Naturally, the beam velocities are assumed to fulfill (58), and the wave frequency is assumed to obey $\omega \ll \omega_{c i} \sim 2 \times 10^{5} \mathrm{~s}^{-1}$. Using Eq. (93) the characteristic time scale for energy conversion then becomes $t \sim \Psi^{-1} \sim 10^{7}\left(\mathcal{E}_{E, \text { ini }}\right)^{-1 / 2} \mathrm{~s}$, where the electromagnetic wave energy density should be given in SI-units. Apparently we need wave energy densities $\mathcal{E}_{E, \text { ini }} \sim 10^{14} \mathrm{~J} / \mathrm{m}^{3}$ to get conversion within 1 second. Note that EMW energy densities in the laboratory can be magnitudes larger than this 29] and the value is also modest as compared to some astrophysical events, like gamma-ray bursts. However, we note that the required wave energy content is still very large, since the EMW must be distibuted in a volume not less than a cube with a side of 1 light second, for efficient GW conversion to take place.

The model we have presented is idealized in several ways, e.g. we have assumed a uniform background and monochromatic waves. The model can be extended to include weakly nonuniform backgrounds, where the interaction can be treated using mode conversion theory, see e.g. Ref. [27] and Refs. therein. It should also be noted that the background drift flow is in general also unstable with respect to other types of perturbations, e.g. ion-accoustic perturbations which typically may have a higher growth rate. With a more realistic velocity distribution among the particles, including a thermal spread, the conditions and growth rates for various instabilities change. It is an open question whether GW-EMW instabilities (of the type presented in this paper) can be the only ones (or the dominating ones) in certain regions of parameter space. We have also treated the gamma factors and the background flow as constants in time. This is not a valid approximation for the long term evolution when the change in energy becomes comparable to the initial energy of the source. An exception to this case is interaction between waves with "moderate" positive energies in the presence of a highly energetic background flow, such that $\gamma \approx\left(1-v_{z}^{2}\right)^{-\frac{1}{2}}$ and $v_{z}$ are approximately 
constant for all times. Variations in $\gamma$ ad $v_{z}$ can, as these occurs in the dispersion relation (57), also affect the frequency of the EMW, thereby invalidate the assumed perfect resonance and thus reducing the coupling strength.

In conclusion, energy transfer between GWs, EMWs and a background plasma flow should occur also in less idealized situations than considered in this paper, but in a more involved way. An improved model that correctly describes the long term evolution of the interaction between a GW, an EMW and the background flow in an inhomogeneous background is a project for future research.
[1] M. E. Gertsenshtein, Zh. Eksp. Teor. Fiz. 41, 113 (1961) [Sov. Phys. JETP, 14, 84 (1962)].

[2] Y. B. Zeldovich, Zh. Eksp. Teor. Fiz. 65, 1311 (1973) [Sov. Phys. JETP, 65, 1311 (1973)].

[3] L. P. Grishchuk and A. G. Polnarev, in General Relativity and Gravitation, edited by A. Held (Plenum, New York, 1980) Vol. 2.

[4] M. Marklund, G. Brodin and P. Dunsby, Astrophys. J. 536, 875 (2000).

[5] G. Brodin, M. Marklund and M. Servin, Phys. Rev. D 63, 124003 (2001);

M. Maggiore, Phys. Rep. 331, 283 (2000);

Yu. G. Ignat'ev, Phys. Lett. A 320, 171 (1997).

[6] M. Servin, G. Brodin, M. Marklund and M. Bradley, Phys. Rev. E 62, 8493 (2000).

[7] D. Papadopoulos, N. Stergioulas, L. Vlahos, et al. A \& A 377, 701 (2001).

[8] R. Bingham et al., Phys. Scr. T75, 61 (1998).

[9] K, Kleidis, H. Varvoglis and D. Papadopoulos, Class. Quantum Grav. 13, 2547 (1996).

[10] J. Weiland and H. Wilhelmsson, Coherent Nonlinear Interaction of Waves in Plasmas (Pergamon Press, New York, 1977);

A. Craik, Wave interactions and fluid flows (Cambridge Univeristy Press 1985).

[11] A. M. Anile, J. K. Hunter and B. Turong, J. Math. Phys. 40, 4474 (1999).

[12] A. Greco and L. Seta, Class. Quantum Grav. 15, 3655 (1998).

[13] P. G. Macedo and H. Nelson, Phys. Rev. D 28, 2328 (1983).

[14] In general, the source terms may contain derivatives on $f_{(n)}$ and $f_{(m)}$. As we restrict ourself to weak interaction these derivatives are later approximated by algebraic expressions, i.e. involving frequencies and wave numbers.

[15] M. Marklund, G. Brodin and P. K. S. Dunsby, Astrophys. J. 536, 875 (2000);

G. Brodin, M. Marklund, P. K. S. Dunsby, Phys. Rev. D 62, 104008 (2000).

[16] R. A. Isaacson, Phys. Rev. 166, 1263 (1968).

[17] T. H. Stix, Waves in Plasmas (American Institite of Physics, New York, 1992);

D. R. Nicholson, Introduction to Plasma Theory (John Wiley \& Sons Inc., 1983).

[18] L. D. Landau and E. M. Lifshitz, The Classical Theory of Fields (Pergamon Press, Oxford, 1975).

[19] F. Chen, Introduction to Plasma Physics and Controlled
Fusion (Plenum Press, New York, 1984).

[20] The dispersion relation, however, depends on the wave amplitude through the gamma factors, but due to the circular polarization the gamma factors are constant (for free waves).

[21] L. Stenflo, Phys. Scr. 14, 320 (1976).

[22] Formally, the drift velocities, $v_{z(i)}$, cannot be taken arbitrarily. They should fulfill $j_{z}=0$ for the given solution to be valid, or else the drifts produce a background magneic field that is not accounted for in the solution. On the other hand, one can always divide a fluid component into several species, each with a different drift velocity and thereby regain freedom in the relative drifts. Thus we discard this restriction because it should not influence the final result qualitatively.

[23] From a mathematical point of view, our large EMW solution (together with the background drift) contains more energy than the state with only the pure drift flow. However, from energy conservation it is clear that the electromagnetic field amplitude cannot be physically altered without simultaneously affecting the drift velocity. When confirming that the wave energy is negative, one should compare with the state where this change in drift flow has been taken into account.

[24] One could expect that the curves in FIG 1. should be even functions of the velocity. The symmetry is, however, broken by the definite propagation direction. The important point is that the relation between the wave polarization and the particle gyration (determined by $\Omega_{c e}$ ) is fixed by the condition $\omega=k$, which depends on the flow velocities, as seen by Eq. (58).

[25] In general also the phase $\phi$ evolves in time. Eqs. (80)(82) are still valid but should be supplemented with an evolution equation for the phase, see e.g. Ref. [10]. For our purposes, however, it suffices to consider the special cases where the phase is constant.

[26] By conversion rate we mean the inverse time scale for one wave to be converted into another type of wave.

[27] R. Littlejohn and W. Flynn, Phys. Rev. Lett. 70, 1799 (1993).

[28] This example makes use of the substitution $\partial_{t} \rightarrow v_{g} \partial_{z}$ appropriate for a boundary value problem. Effects of an inhomogeneous background is neglected.

[29] G. A. Morou, C.P.J. Barty and M.D. Perry, Phys. Today, 51, No 1, 22 (1998). 